\title{
LAS ELECCIONES CANTONALES EN FRANCIA
}

Al igual que en las otras elecciones francesas, en las cantonales se sigue el sistema de escrutinio uninominal mayoritario a dos vueltas. De esta forma, con la sola excepción de París (que es a la vez municipio y departamento y donde los consejeros municipales son consejeros generales), los días 14 y 21 de marzo más de la mitad del electorado francés acudió a las urnas para elegir 1.945 consejeros generales por un mandato de seis años.

Hasta ahora, las elecciones cantonales (el cantón - formado por un municipio o agrupación de municipios- no es una colectividad territorial, sino una circunscripción electoral en la que se elige un consejero general) tenían un carácter más administrativo que político; sin embargo, estas últimas han estado fuertemente politizadas, principalmente por dos motivos: primero, porque, después de las elecciones presidenciales y legislativas del pasado año, ésta era la primera ocasión que tenía el electorado francés de ratificar o rectificar su veredicto, y segundo, porque, una vez celebradas, entraría en vigor la «Ley relativa a los derechos y libertades de los municipios, departamentos y regiones», es decir, una ley de descentralización, que concede a los presidentes de los Consejos Generales poderes que hasta este momento correspondían exclusivamente a los prefectos.

Los resultados de la primera vuelta (celebrada el domingo 14 de marzo) concedieron una cierta ventaja a la oposición, ventaja que fue mantenida en la segunda vuelta, que se celebró siete días más tarde y en la que se ratificó el nuevo rumbo del electorado francés, concediendo a la oposición la mayoría de puestos en 58 departamentos, mientras que la izquierda sólo dominaba en 35 Consejos Generales.

La derrota de la izquierda en estas cantonales ha sido interpretada fundamentalmente en base a dos razones: la primera, el hecho de que los franceses no desean ese radical cambio de sociedad, que es el caballo de batalla socialista, y la segunda, el sentimiento, todavía difuso, pero cristalizante, de que diez meses después del 10 de mayo el poder socialista ha arrastrado a un proceso político, social y económico que conduce inexorablemente a la decadencia de Francia.

Los comentarios de los dirigentes de la oposición tras la ligera ventaja obtenida sobre la izquierda pueden resumirse en estas palabras del presidente del RPR, Jacques Chirac: «Los franceses han confirmado los resultados de la primera vuelta manifestando su decepción y su inquietud por el porvenir a la vista de la política seguida por el Gobierno. La oposición ha confirmado su éxito. Los resultados de estas elecciones marcan la evolución de nuestros ciudadanos. E1 Gobierno deberá tenerlo en cuenta si quiere ser realmente digno de una democracia que no infravalora la calidad del escrutinio popular» (Le Monde, 23-3-82). 
Por el contrario, en los cuarteles del partido gubernamental los resultados de las cantonales no se interpretaba como una derrota irreversible: «... ¿Por qué estos resultados? Porque ha habido un reflejo mecánico. La izquierda ha ido muy lejos en las últimas elecciones legislativas como consecuencia de una dinámica creada por la elección presidencial. Lógicamente, la derecha se ha movilizado más que la izquierda al ver en estas elecciones una revancha política, una postura nacional» (Le Monde, 23-3-82, entrevista a Lionel Jospin, primer secretario del Partido Socialista francés).

Así, pues, si bien la izquierda detenta el poder ejecutivo y tiene la mayoría en la Asamblea Nacional, los resultados electorales de marzo pasado han revelado que la mayoría de la izquierda ha perdido en gran medida la confianza de muchos franceses; es decir, parafraseando a F. Mitterrand, que la mayoría sociológica ha dado un aldabonazo en las puertas del poder.

BALANCE DE LAS DOS VUELTAS EN LA METROPOLI, EN PUESTOS

\begin{tabular}{|c|c|c|c|}
\hline PARTIDOS & Salientes & Elegidos & Balance \\
\hline Extrema Izquierda $\ldots \ldots \ldots$ & 2 & 1 & -1 \\
\hline P.C.F. $\ldots \ldots \ldots \ldots$ & 236 & 191 & -45 \\
\hline P. $S . \ldots \ldots \ldots \ldots \ldots$ & 511 & 504 & -7 \\
\hline M. R. G. $\ldots \ldots \ldots \ldots \ldots \ldots$ & 88 & 61 & -27 \\
\hline Otros partidos de izquierda. & 61 & 41 & -20 \\
\hline R.P.R. $\ldots \begin{array}{llllll} & \ldots & \ldots & \ldots & \ldots & \ldots\end{array}$ & 179 & 323 & +144 \\
\hline U.D.F. $\ldots \begin{array}{lllll} & \ldots & \ldots & \ldots & \ldots\end{array}$ & 389 & 460 & +71 \\
\hline Otros partidos de derecha. & 311 & 363 & +52 \\
\hline Extrema Derecha $\ldots \ldots \ldots$ & 1 & 1 & - \\
\hline TOTAL $\ldots \ldots \ldots \ldots$ & 1.778 & 1.945 & - \\
\hline
\end{tabular}

Indice de siglas:

P. C. F.: Partido Comunista Francés.

P. S.: Partido Socialista.

M. R. G.: Movimiento de los Radicales de Izquierda.

R. P. R.: Reagrupamiento por la República (gaullistas).

U. D. F.: Unión por la Democracia Francesa (giscardianos).

FUENTE: Ministère de 1'Intérieur Français.

RESULTADOS DE LAS ELECCIONES CANTONALES EN LA PRIMERA Y SEGUNDA VUELTA (PORCENTAJE)

\begin{tabular}{|c|c|c|}
\hline PARTIDOS & 1.a vuelta & 2. ${ }^{\mathrm{a}}$ vuelta \\
\hline P.C.F. ... ... . & 15,9 & 13,66 \\
\hline P. S. у M. R. G. $\ldots$... & 31,60 & 36,80 \\
\hline Otros partidos de izquierda. & 2,1 & 1,38 \\
\hline P. R.P. (gaullistas) $\ldots \quad \ldots$ & 18 & 22,51 \\
\hline U.D.F. (giscardianos) $\ldots$ & 18,8 & 15,54 \\
\hline Otros partidos de derecha ... & 13,1 & 10,03 \\
\hline Ecologistas $\ldots \ldots \ldots \ldots$ & 0,4 & 0,6 \\
\hline
\end{tabular}

FuENTE: Ministère de l'Intérieur Français. 
BALANCE DE PUESTOS OBTENIDOS (METROPOLI Y ULTRAMAR)

\begin{tabular}{|c|c|c|c|c|c|}
\hline PARTIDOS & $\begin{array}{c}\text { Puestos } \\
\text { renovados }\end{array}$ & $\begin{array}{l}\text { Elegidos } \\
\text { 1. }{ }^{\mathrm{a}} \text { vuelta }\end{array}$ & $\begin{array}{c}\text { Elegidos } \\
2 .{ }^{a} \text { vuelta }\end{array}$ & $\begin{array}{l}\text { Total } \\
\text { elegidos }\end{array}$ & Balance \\
\hline Extrema Izquierda $\ldots \ldots \ldots$ & 2 & 4 & 0 & 4 & + \\
\hline P. C. F. ... $\ldots \ldots \ldots \ldots \ldots$ & $24 \overline{4}$ & 26 & 173 & 199 & -45 \\
\hline $\begin{array}{llllllll}\text { P. S. } & \ldots & \ldots & \ldots & \ldots & \ldots & \ldots & \ldots\end{array}$ & 525 & 121 & 394 & 515 & -10 \\
\hline M. R. G. $\ldots \ldots \ldots \ldots \ldots \ldots$ & 87 & 17 & 42 & 59 & -28 \\
\hline Otros partidos de izquierda. & 56 & 17 & 22 & 39 & -17 \\
\hline TOTAL IZQUIERDA $\ldots \ldots$ & 914 & 185 & 631 & 816 & -98 \\
\hline U. D. F.M. D. S.* $\ldots \ldots \ldots$ & 13 & 11 & 0 & 11 & - \\
\hline 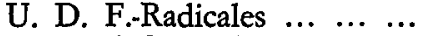 & 54 & 37 & 13 & 50 & - \\
\hline U. D. F.-C. D. S.* $\ldots \ldots \ldots$ & 147 & 136 & 47 & 183 & +36 \\
\hline U. D. F.P. R.* ${ }^{*} \ldots \ldots \ldots$ & 158 & 146 & 61 & 207 & +49 \\
\hline U. D. F. y otros $\ldots \ldots \ldots \ldots$ & 94 & 59 & 45 & 104 & +10 \\
\hline R. P. R. $\begin{array}{lllllll} & \ldots & \ldots & \ldots & \ldots & \ldots & \ldots\end{array}$ & 199 & 196 & 152 & 348 & +149 \\
\hline $\begin{array}{llllllll}\text { Moderados } & \ldots & \ldots & \ldots & \ldots & \ldots\end{array}$ & 247 & 167 & 111 & 278 & +31 \\
\hline Total Derecha $\ldots \ldots \ldots$ & 912 & 752 & 429 & 1.181 & +264 \\
\hline
\end{tabular}

- M. D. S.: Movimiento Socialdemócrata.

- P. R.: Partido Republicano.

* C. D. S.: Confederación Demócrata-Social.

FuENTE: Datos suministrados por Le Monde de 23-3-82.

\section{LA ABSTENCION EN FRANCIA DESDE 1976 (\%)}

\begin{tabular}{|c|c|c|c|c|c|c|}
\hline \multirow{7}{*}{$\begin{array}{llllll}\text { Marzo } & 1976 & \ldots & \ldots & \ldots & \ldots \\
\text { Marzo } & 1978 & \ldots & \ldots & \ldots & \ldots \\
\text { Marzo } & 1979 & \ldots & \ldots & \ldots & \ldots \\
\text { Abril-mayo } & 1981 & \ldots & \ldots & \ldots \\
\text { Junio } & 1981 & \ldots & \ldots & \ldots & \ldots \\
\text { Marzo } & 1982 & \ldots & \ldots & \ldots & \ldots\end{array}$} & \multicolumn{2}{|c|}{ Cantonales } & \multicolumn{2}{|c|}{ Legislativas } & \multicolumn{2}{|c|}{ Presidenciales } \\
\hline & 34,7 & 32,3 & - & - & - & - \\
\hline & - & - & 16,6 & 15,3 & - & - \\
\hline & 34,5 & 34,6 & - & - & - & - \\
\hline & - & - & - & - & 18,9 & 14,1 \\
\hline & 一 & - & 29,6 & 25,5 & - & - \\
\hline & 31,8 & 29,7 & - & - & - & - \\
\hline
\end{tabular}

FuENTE: Ministère de 1'Intérieur Français.

Pilar Mellado Prado 